\title{
Erratum: Entanglement structure of the Hubbard model in momentum space [Phys. Rev. B 92, 235116 (2015)]
}

\author{
G. Ehlers, J. Sólyom, Ö. Legeza, and R. M. Noack
}

(Received 5 July 2017; published 27 July 2017)

DOI: 10.1103/PhysRevB.96.039906

The legends in the original Fig. 6 mislabeled the curves. As indicated in the corrected version shown here, the triangles depict the doped case $(n=0.722)$, and the square symbols depict the half-filled case $(n=1.0)$. The description of the figure in the text is correct, and this error does not affect the other results or the conclusions of the paper.

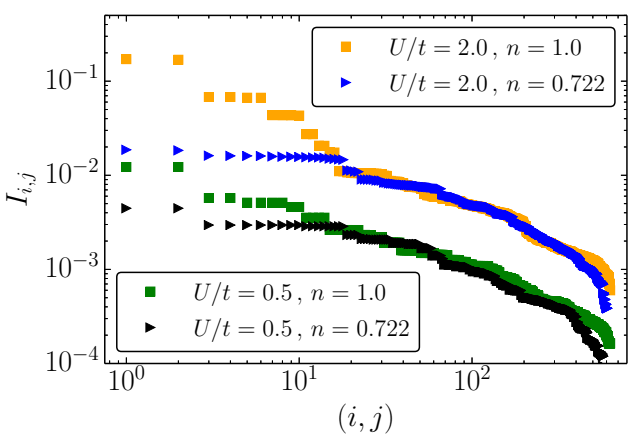

FIG. 6. Two-site mutual information $I_{i, j}$ for the ground state of the $6 \times 6$ Hubbard model in momentum space for different $U$ values and different fillings $n$. The values are sorted in descending order. We include only unique entries of $I_{i, j}$, i.e., take $i>j$. 